\title{
Rapid detection of multidrug-resistant tuberculosis
}

\author{
M. Goyal*, R.J. Shaw*, D.K. Banerjee**, R.J. Coker*+, \\ B.D. Robertson ${ }^{++}$, D.B. Young ${ }^{++}$
}

Rapid detection of multidrug-resistant tuberculosis. M. Goyal, R.J. Shaw, D.K. Banerjee, R.J. Coker, B.D. Robertson, D.B. Young. CERS Journals Ltd 1997.

ABSTRACT: Transmission of multidrug-resistant strains of Mycobacterium tuberculosis (MDR-TB) presents a serious problem for infection control in hospitals, particularly in the context of co-infection with the human immunodeficiency virus (HIV). We report on the use of molecular genetic tools to allow rapid assessment of samples from patients potentially infected with MDR-TB.

Sputum and bronchoalveolar lavage samples were obtained from two HIV-positive patients with suspected tuberculosis, who had previous contact with a known MDR-TB index case. Polymerase chain reaction (PCR) was used directly on clinical samples to amplify genetic loci associated with rifampicin resistance $(r p o B)$, and strain-specific polymorphisms (the direct repeat (DR) region). Drug resistance was determined using a commercially available kit for detection of point mutations in the rpoB gene (Inno-Lipa RifTB; Innogenetics, Belgium), and confirmed by nucleotide sequencing. Strain variation was determined using the spoligotyping method, based on the presence or absence of variable linker sequences within the DR region.

In one patient, infection with a MDR strain identical to that of a known index case was demonstrated. A second patient, although positive for M. tuberculosis, was found to be infected with a rifampicin-sensitive strain. Results were obtained within $48 \mathrm{~h}$, allowing appropriate treatment to be initiated and infection control measures to be implemented.

PCR-based tests for strain-typing and for identification of rifampicin resistance provide important tools for identifying patients with MDR-TB and for rapid monitoring of potential nosocomial spread of MDR-TB. Prompt confirmation or exclusion of possible transmission allows early clinical intervention to prevent future outbreaks of multidrug-resistant $M$. tuberculosis.

Eur Respir J 1997; 10: 1120-1124.
Depts of *Respiratory Medicine, ${ }^{++}$Medical Microbiology and ${ }^{+}$GenitoUrinary Medicine, Imperial College School of Medicine at St Mary's, London, UK. **Dept of Medical Microbiology, St George's Hospital Medical School, London, UK

\section{Correspondence: M. Goyal}

Dept of Respiratory Medicine

Imperial College School of Medicine at St Mary's

Norfolk Place

London W2 1PG

UK

\section{Keywords: Direct repeat}

multidrug-resistant tuberculosis polymerase chain reaction

rifampicin

rров gene

spoligotyping

Received: October 311996

Accepted after revision February 281997

This work was supported by the British Lung Foundation.
Strains of Mycobacterium tuberculosis resistant to frontline drugs, including isoniazid and rifampicin, (multi-drug-resistant $M$. tuberculosis (MDR-TB)) have been responsible for a series of frequently fatal disease outbreaks [1-3]. Outbreaks have generally been associated with nosocomial transmission in hospital or prison settings, often including, though not restricted to, patients co-infected with human immunodeficiency virus (HIV) [3-7]. Following outbreaks in the United States in the early 1990s, an extensive research effort has been invested in the design of improved procedures for infection control, and in development of tools for rapid detection of drug-resistant isolates [8, 9].

Faced with the slow growth rate of $M$. tuberculosis, it takes weeks or even months to identify drug-resistant strains by conventional microbiology. With the prospect of developing rapid deoxyribonucleic (DNA)-based tests for resistance, investigators have focused on identification of the genetic changes responsible for resistance [8]. For each of the isolates analysed in detail, drug-resistance in M. tuberculosis has been mediated by changes affecting chromosomal genes, with no evidence of in- volvement of any transmissible genetic elements. Multidrug resistance is built-up by an accumulation of individual mutations conferring resistance to single drugs [10]. Mutations at several different loci can give rise to isoniazid resistance $[11,12]$, but resistance to rifampicin is almost always mediated by mutation of residues within a short stretch of the gene encoding the $\beta$-subunit of ribonucleic acid (RNA) polymerase (rpoB) [13, 14]. Rifampicin resistance is often associated with resistance to isoniazid, and is considered a useful surrogate marker for MDR-TB [8]. In the present study, we have used a commercially available kit (Inno-Lipa RifTB; Innogenetics, Belgium) for rapid detection of $r p o B$ mutations associated with rifampicin resistance.

DNA-fingerprinting based on variations in the pattern of IS6110 insertions has been widely used as an epidemiological tool in monitoring patterns of transmission of $M$. tuberculosis, including analysis of MDR outbreaks $[15,16]$. Bacterial cultures are required for this type of analysis, however, and results are essentially retrospective. In order to allow tracking of mycobacterial strains within a clinically useful timeframe, we have 
used a polymerase chain reaction (PCR)-based typing system referred to as "spoligotyping". This technique is based on amplification of a region of the $M$. tuberculosis genome containing a series of direct repeat elements interspersed by variable spacer sequences [17]. PCR products are subsequently analysed by hybridization to membrane-bound oligonucleotides corresponding to the variable spacers, different isolates being characterized by their pattern of hybridization to differing sets of spacers. The level of strain discrimination provided by spoligotyping is slightly less than that of IS6110 fingerprinting, but its direct application to clinical samples represents a significant advantage when rapid results are required [18].

We report on the combined use of molecular tests for rifampicin resistance and strain-typing to facilitate rapid intervention and control of transmission of MDR-TB.

\section{Materials and methods}

\section{Patients and clinical samples}

Samples were obtained from three patients. The isolates from patient No. 1, the index case of tuberculosis, were resistant to rifampicin, isoniazid and pyrazinamide, as well as to cycloserine, rifabutin, clofazimine and ciprofloxacin. A sputum sample obtained from patient No. 2, an HIV-positive male and a contact of patient No. 1 was obtained on July 4, 1996 and stained weakly positive for acid-fast bacteria. Patient No. 3, an HIV-positive male, had also been exposed to MDR-TB from this outbreak. He subsequently developed a fever and unproductive cough, and bronchoalveolar lavage (BAL) was performed. No acid-fast bacteria were found in the BAL sample. A bacterial culture from an epidemiologically unrelated case of tuberculosis, patient No. 4, was also included in the study.

Clinical specimens (sputum and BAL) were digested and decontaminated by the N-acetyl-L-cysteine (NAC) $2 \% \mathrm{NaOH}$ method. The samples were concentrated and the pellet resuspended in $100 \mu \mathrm{L}$ of sample buffer (Trisethylenediamine tetra-acetic acid (EDTA) (TE buffer), $\mathrm{pH}$ 8.0), and then placed in a heat block at $80^{\circ} \mathrm{C}$ for 20 min. DNA was extracted from the treated clinical samples and bacterial cultures by the method described by vAN SoOLINGEn et al. [19]. Briefly, organisms were lysed using a cocktail of lysozyme (final concentration 1 $\left.\mathrm{mg} \cdot \mathrm{mL}^{-1}\right)$, proteinase $\mathrm{K}\left(10 \mathrm{mg} \cdot \mathrm{mL}^{-1}\right)$ and Sodium dodecyl sulphate (SDS) (10\%), followed by treatment with $\mathrm{N}$-acetyl-N,N,N-trimethyl ammonium bromide. DNA was prepared from the supernatants by phenol chloroform extraction and ethanol precipitation.

\section{Detection of rifampicin resistance}

A portion of the $r p o B$ gene was amplified from DNA preparations by nested PCR, using primers provided as part of the Inno-Lipa RifTB kit according to the instructions provided by the manufacturers (Innogenetics, Belgium). Resulting biotinylated PCR products were hybridized with oligonucleotide probes immobilized as parallel lines on membrane strips. The oligonucleotides included five probes corresponding to sequences present in the wild-type, rifampicin-sensitive, gene (S1-S5), together with four probes corresponding to the most frequently observed resistance mutations (R2, R4a, R4b and R5). After hybridization and washing according to the manufacturer's protocol, streptavidin labelled with alkaline phosphatase was added to detect bound biotinylated hybrids. Binding was visualized as a purple precipitate following incubation with 5-bromo-4-chloro-3-indolyl phosphate (BCIP) chromogen. At the same time, the initial 450 base pair (bp) PCR products were also characterized by sequence analysis of both strands, using an ABI 310 Genetic Analyser (Perkin Elmer, Applied Biosystem Division, Warrington, Cheshire.). Sequencing reactions were performed with the ABI PRISM Dye Terminator cycle sequencing Ready Reaction Kit (Perkin Elmer), using the internal oligonucleotides as primers. Data were analysed using the SeqEd Software (Perkin Elmer).

\section{Strain-typing (spoligotyping)}

The direct repeat (DR) region from each sample was amplified by PCR, using the primers:

\section{5' CCAAGAGGGGACGGAAAC 3' and 5' GGTTTTGGGTCTGACGAC 3' (biotinylated at 5 ' end)}

The conditions used for PCR were 3 min incubation at $96^{\circ} \mathrm{C}$, followed by 20 cycles of $1 \mathrm{~min}$ at $96^{\circ} \mathrm{C}, 1 \mathrm{~min}$ at $55^{\circ} \mathrm{C}$, and $30 \mathrm{~s}$ at $72^{\circ} \mathrm{C}$. PCR products $(20 \mu \mathrm{L}$ in 150 $\mu \mathrm{L}$ of $2 \times$ saline sodium phosphate EDTA (SSPE), supplemented with $0.1 \%$ SDS) were heat denatured for 10 min at $100^{\circ} \mathrm{C}$, and then hybridized for $60 \mathrm{~min}$ at $55^{\circ} \mathrm{C}$ to a Biodyne $\mathrm{C}$ membrane with immobilized oligonucleotides corresponding to spacer sequences (provided by J. van Embden, Bilthoven, The Netherlands). Spacer oligonucleotide sequences were derived from the DR regions of $M$. tuberculosis $\mathrm{H} 37 \mathrm{Rv}$, and Mycobacterium bovis bacille Calmette-Guérin (BCG). The filter was washed with $2 \times \mathrm{SSPE}$ (supplemented with $0.5 \%$ SDS) for $10 \mathrm{~min}$ at $55^{\circ} \mathrm{C}$, and then incubated in streptavidinperoxidase conjugate (Boehringer, Mannheim; $2.5 \mu \mathrm{L}$ in $10 \mathrm{~mL}$ of $2 \times \mathrm{SSPE}$, supplemented with $0.5 \%$ SDS) for $45 \mathrm{~min}$ at $42^{\circ} \mathrm{C}$. The membrane was washed and hybridizing DNA was detected using enhanced chemiluminescence (ECL) detection liquid (Amersham, UK) and exposure of the membrane to X-ray film for $5 \mathrm{~min}$ (Hyperfilm, Amersham).

\section{Results}

Five samples were analysed for the presence of mutations associated with rifampicin resistance. These included: two independent bacterial cultures from the index case (patient No. 1); a smear-positive sputum from patient No. 2; a smear-negative BAL from patient No. 3; and a bacterial culture from patient No. 4 . Using the Inno-Lipa RifTB test, samples from patients Nos. 1 and 2 both showed loss of binding to oligonucleotide S5, along with 


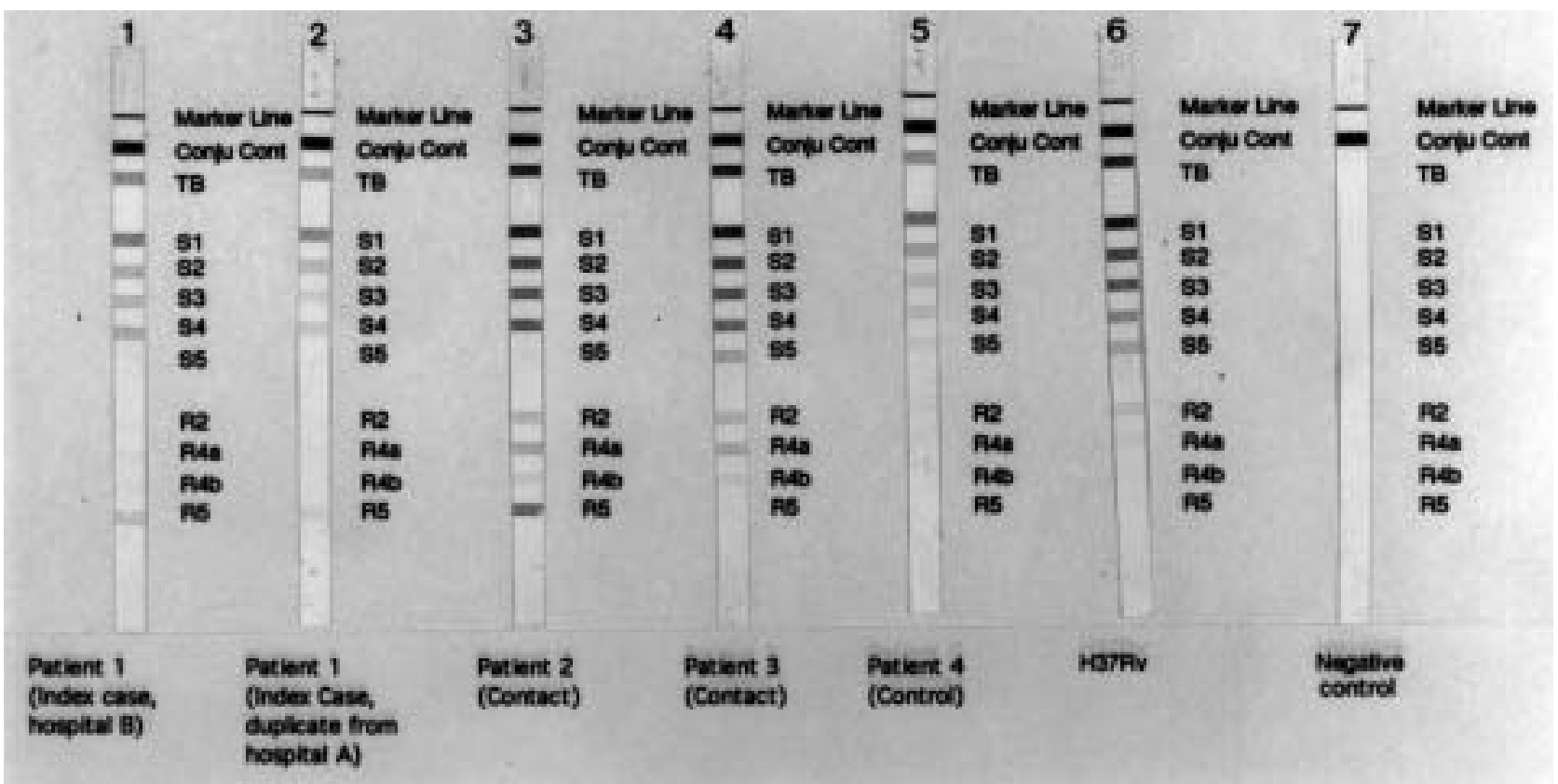

Fig. 1. - Analysis of rifampicin resistance using the Inno-Lipa RifTB test (Innogenetics, Belgium). A fragment of the rpoB gene was amplified from bacterial cultures (patients Nos. 1 and 4), or clinical samples (patients Nos. 2 and 3), and tested for mutations by hybridization to membrane-bound oligonucleotides corresponding to sensitive (S1-S5) and resistant alleles (R2, 4a, 4b and 5). The hybridization pattern of isolates from patients Nos. 1 and 2 (lanes 1-3) indicated mutations in the region corresponding to amino acids 529-533. Sequence analysis confirmed the presence of a point mutation in codon 531. Isolates from patients Nos. 3 and 4 (lanes 4 and 5) had wild-type rpoB sequences. Lane 6 shows the hybridization pattern obtained using the rifampicin-sensitive laboratory strain, M. tuberculosis H37Rv. Lane 7 is a control without deoxribonucleic acid (DNA).

positive binding to R5 (fig. 1). These two probes cover the region of the rpoB gene encoding amino acids 529533 , with the binding pattern being consistent with that of a resistant allele. Sequence analysis confirmed the presence of an identical substitution of thymidine (T) for cytosine $(\mathrm{C})$ within codon 531 in each case, resulting in exchange of leucine (TTG) for serine (TCG) in the amino acid sequence derived. The corresponding $r p o B$ fragment was similarly amplified from the smearnegative BAL sample from patient No. 3. Results from the Inno-Lipa RifTB test (fig. 1, lane 4), and from DNA sequence analysis, identified this as the wild-type rifampicin-sensitive allele. Similarly, the PCR product from an unrelated $M$. tuberculosis isolate (patient No. 4) was confirmed as rifampicin-sensitive (fig. 1, lane 5).

M. tuberculosis isolates from patients Nos. 1, 2 and 4 were also characterized by spoligotyping (fig. 2). In this assay, the single round of PCR amplification did not generate a product from the smear-negative BAL sample provided for patient No. 3. The results demonstrated an identical spoligotype for isolates from patients Nos. 1 and 2, again consistent with nosocomial transmission.
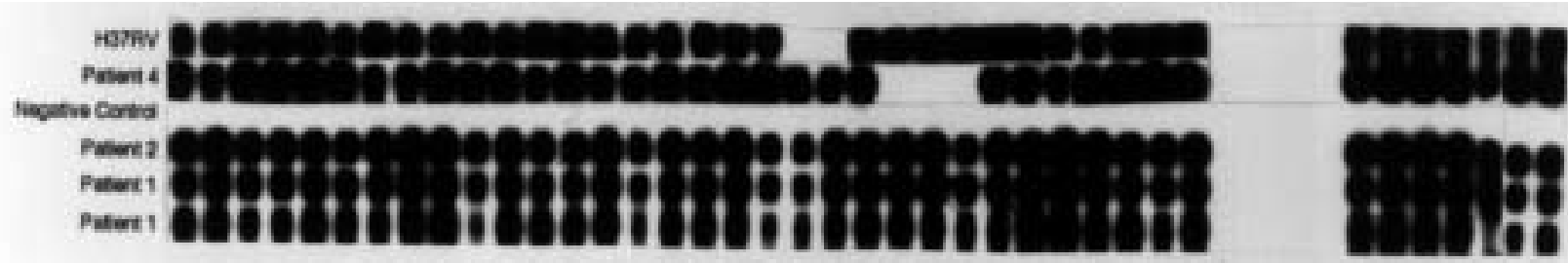

Fig. 2. - Spoligotype analysis of M. tuberculosis isolates. Isolates from patients Nos. 1 (lanes 5 and 6), 2 (lane 4 ) and 4 (lane 2 ) were compared by spoligotyping. Patients Nos. 1 and 2 were found to be infected with identical isolates; patient No. 4 was epidemiologically unrelated and was infected with a different strain type. Lane 1 shows the spoligotype pattern for M. tuberculosis H37Rv. Lane 3 is a control without deoxyribonucleic acid (DNA).

Table 1. - Characteristics of strains isolated from different patients

\begin{tabular}{|c|c|c|c|c|}
\hline $\begin{array}{l}\mathrm{Pt} \\
\text { no. }\end{array}$ & $\begin{array}{l}\text { Contact with } \\
\text { MDR-TB }\end{array}$ & Sample & $r p o B$ sequence & $\begin{array}{c}\text { Spoligotype } \\
\text { pattern }\end{array}$ \\
\hline 1 & Index case & Bacterial culture & Ser $^{531 \rightarrow \text { Leu }}$ & A \\
\hline 2 & Yes & Sputum (smear-positive) & Ser $^{531} \rightarrow$ Leu & A \\
\hline 3 & Yes & BAL (smear-negative) & Wild-type & ND \\
\hline 4 & No & Bacterial culture & Wild-type & $\mathrm{B}$ \\
\hline
\end{tabular}

ND: not determined; MDR-TB: multidrug-resistant tuberculosis; Pt: patient; BAL: bronchoalveolar lavage; Leu: leucine; Ser: serine. 
Table 1 summarizes the results of the molecular analysis. Patient No. 2 was placed on a drug regimen consisting of a combination of amikacin, cycloserine, prothionamide, ofloxacin and ethambutol, which was changed on August 19, 1996 to amikacin, cycloserine, ethambutol, pyrazinamide and clarithromycin, and was accommodated in an isolation facility. The sputum sample from patient No. 2 subsequently grew M. tuberculosis after 6 weeks, which was found after another 4 weeks to be resistant to rifampicin, isoniazid, streptomycin and ethambutol, and sensitive to ethionamide, cycloserine, capreomycin, period-acid-Schiff (PAS), amikacin and pyrazinamide. Patient No. 3 was being treated and responded to conventional therapy (rifampicin, isomiazid and pyrazinamide) as an out-patient. No growth was observed from the BAL sample from patient No. 3 by 12 weeks of culture. However, the sample was found by an independent laboratory to be positive for $M$. tuberculosis using the Gen-Probe method.

\section{Discussion}

This study was conducted following requests from clinicians for a rapid assessment of the possibility of transmission from a known MDR-TB index case. Based on strain-typing and analysis of resistance-associated mutations, the present results strongly suggested that patient No. 2 had, in fact, been infected with the same strain as the index case, while patient No. 3 had probably acquired an unrelated infection with a rifampicinsensitive strain. A key feature of the present study is that a sputum sample from patient No. 2 was collected on July 4, 1996 and transferred after the weekend to our laboratory, where it arrived on the evening of July 9,1996 , and we were able to relay detailed results to the clinician on July 12, 1996, thus allowing initiation of appropriate treatment, and implementation of infection control measures to limit any further spread. Furthermore, rapid detection of tuberculosis and clarification of sensitivity allowed conventional treatment to be initiated early for patient No. 3, with early discharge from hospital. The speed of this new approach contrasts with conventional culture and sensitivity testing, the results of which were still awaited at the time of submission of this manuscript.

The Inno-Lipa RifTB test provides a relatively simple assay for detection of mutations associated with rifampicin resistance and, as demonstrated here, the nested PCR format provides a sensitive detection system capable of generating a signal even in a smear-negative sample. Studies of the reproducibility of the Inno-Lipa RifTB on a large number of extracts from sputum have yet to be completed and, therefore, in the present study we have combined this method with automated DNA sequencing. A potential drawback of this approach is that it will only detect the set of mutations already defined in the $r p o B$ gene, and may, therefore, fail to identify any novel resistant alleles. However, a series of studies have identified $r p o B$ mutations in $>97 \%$ of more than 200 rifampicin-resistant isolates of M. tuberculosis, with mutation of codon 531, as observed in the present study, the most frequent alteration [8]. Combination of $r p o B$ analysis with a PCR-based typing system provides a powerful approach to monitoring the transmission of MDR-TB. Detection of rifampicin resistance by molecular biology techniques is considerably more straightforward than detection of resistance to other drugs. Although the final choice of antimycobacterial agents used to treat the patient requires full knowledge of the drug sensitivity patterns of the organisms, rifampicin resistance rarely occurs in isolation and serves as a good surrogate marker for multidrug resistance.

The spoligotyping system for strain-typing is fast and reliable, generating data that can be readily analysed by eye, and stored using any simple word-processing programme. Strain-typing itself does not provide direct information about drug susceptibility, but a register of spoligotypes from known MDR isolates may be particularly useful in providing early warning of possible infection with these strains. Whilst previous MDR-TB outbreaks have been characterized using IS6110 fingerprinting [20], there are considerable clinical advantages in having a test which, when applied to smear-positive sputum, can provide strain information within one or two days. It is during this period, and in these potentially infectious patients, that crucial decisions have to be made in relation to treatment regimens and appropriate patient accommodation.

In the present study, spoligotyping was possible in the smear-positive samples but not in the smear-negative samples. Other studies on several hundred sputum samples (unpublished) suggest that spoligotyping can provide typing data on smear-positive sputum samples. As with any PCR-based technique, the test has lower sensitivity in smear-negative disease. Other PCR-based typing techniques which have been developed include: arbitrarily primed (AP) PCR [21], random amplified polymorphic DNA analysis (RAPD) [22, 23], and mixed linker [24]. A less complex PCR-based typing method was outlined by Ross and DwYER [25]. They used primers that bind to the ends of IS6110 to amplify DNA between closely spaced copies of this element. PLIKAYTIS et al. [26] combined multiple polymorphic tandem repeat (MPTR) and IS6110 to develop the IS6110 ampliprinting typing method. Another method exploited the variability in KatG gene [27]. The method called "double repetitive element PCR" (DRE-PCR) utilized the amplification of $M$. tuberculosis DNA segments located between two copies of repetitive elements IS6110 and the polymorphic guanine-cytosine rich repetitive sequence (PGRS) [28]. Recently, another PCR-based method called "hemi-nested inverse PCR" for IS6110 has also been developed [29]. There is little evidence to date that these PCR-based techniques can be used routinely on sputum samples.

Initial outbreaks of MDR-TB in the United States were associated with high numbers of fatalities amongst patients and health care workers. Use of molecular tests for rifampicin resistance and strain-typing to detect transmission of multidrug-resistant tuberculosis will facilitate the rapid response required to limit the extent and severity of future outbreaks.

Acknowledgement: The authors are grateful to J. van Embden for assistance in establishing the spoligotyping assay. 


\section{References}

1. Snider D Jr, Roper WL. The new tuberculosis. $N$ Engl J Med 1992; 326: 703-705.

2. Frieden TR, Sterling T, Pablos-Mendez A, Kilburn JO, Cauthen GM, Dooley SW. The emergence of drug-resistant tuberculosis in New York City. N Engl J Med 1993; 328: 521-526.

3. Anon. Multidrug-resistant tuberculosis outbreak on an HIV ward, Madrid, Spain, 1991-1995. MMWR Morb Mortal Wkly Rep 1996; 45: 330-333.

4. Beck-Sague C, Dooley SW, Hutton MD, et al. Hospital outbreak of multidrug-resistant Mycobacterium tuberculosis infections: factors in transmission to staff and HIV-infected patients. JAMA 1992; 268: 1280-1286.

5. Dooley SW, Villarino ME, Lawrence M, et al. Nosocomial transmission of tuberculosis in a hospital unit for HIVinfected patients. JAMA 1992; 267: 2632-2634.

6. Pearson ML, Jereb JA, Frieden TR, et al. Nosocomial transmission of multidrug-resistant Mycobacterium tuberculosis: a risk to patients and health care workers. Ann Intern Med 1992; 117: 191-196.

7. Valway SE, Greifinger RB, Papania M, et al. Multidrugresistant tuberculosis in the New York State prison system, 1990-1991. J Infect Dis 1994; 170: 151-156.

8. Cole ST, Telenti A. Drug resistance in Mycobacterium tuberculosis. Eur Respir J 1994; 20: (Suppl.) 701s-713s.

9. Wenger PN, Otten J, Breeden A, Orfas D, Beck-Sague CM, Jarvis WR. Control of nosocomial transmission of multidrug-resistant Mycobacterium tuberculosis among healthcare workers and HIV-infected patients. Lancet 1995; 345: 235-240.

10. Heym B, Honore N, Truffot-Pernot C, et al. Implications of multidrug resistance for the future of short-course chemotherapy of tuberculosis: a molecular study. Lancet 1994; 344: 293-298.

11. Banerjee A, Dubnau E, Quemard A, et al. inhA, a gene encoding a target for isoniazid and ethionamide in Mycobacterium tuberculosis. Science 1994; 263: 227230.

12. Zhang Y, Heym B, Allen B, Young D, Cole S. The catalase-peroxidase gene and isoniazid resistance of Mycobacterium tuberculosis. Nature 1992; 358: 591-593.

13. Telenti A, Imboden P, Marchesi F, et al. Detection of rifampicin-resistant mutations in Mycobacterium tuberculosis. Lancet 1993; 341: 647-650.

14. Kapur V, Li LL, Iordanescu S, et al. Characterization by automated DNA sequencing of mutations in the gene $(r p o B)$ encoding the RNA polymerase beta subunit in rifampin-resistant Mycobacterium tuberculosis strains from New York City and Texas. J Clin Microbiol 1994; 32: 1095-1098.

15. Alland D, Kalkut GE, Moss AR, et al. Transmission of tuberculosis in New York City: an analysis by DNAfingerprinting and conventional epidemiologic methods. $N$ Engl J Med 1994; 330: 1710-1716.

16. van Embden JD, Cave MD, Crawford JT, et al. Strain identification of Mycobacterium tuberculosis by DNA fingerprinting: recommendations for a standardized methodology. J Clin Microbiol 1993; 31: 406-409.

17. Groenen PM, Bunschoten AE, van Soolingen D, van Embden JD. Nature of DNA polymorphism in the direct repeat cluster of Mycobacterium tuberculosis: application for strain differentiation by a novel typing method. Mol Microbiol 1993; 10: 1057-1065.

18. Goyal M, van Embden JDA, Young DB, Shaw RJ. Evaluation of spoligotyping as an epidemiological test in three different outbreaks of tuberculosis. Thorax 1995; 50 (Suppl. 2): A35.

19. van Soolingen D, de-Haas PE, Hermans PW, Groenen PM, van Embden JD. Comparison of various repetitive DNA elements as genetic markers for strain differentiation and epidemiology of Mycobacterium tuberculosis. J Clin Microbiol 1993; 31: 1987-1995.

20. Coronado VG, Beck-Sague CM, Hutton MD, et al. Transmission of multidrug-resistant Mycobacterium tuberculosis among persons with human immunodeficiency virus infection in an urban hospital: epidemiologic and restriction fragment length polymorphism analysis. J Infect Dis 1993; 168: 1052-1055.

21. Palittapongarnpim P, Chomyc S, Fanning A, Kunimoto D. DNA fingerprinting of Mycobacterium tuberculosis isolates by ligation mediated polymerase chain reaction. Nucleic Acids Res 1993; 21: 761-762.

22. Linton CJ, Jalal H, Leeming JP, Miller MR. Rapid discrimination of Mycobacterium tuberculosis strains by random amplified polymorphic DNA analysis. J Clin Microbiol 1994; 32: 2169-2174.

23. Abed Y. Davin-Regli A, Bollet C, De Micco P. Efficient discrimination of Mycobacterium tuberculosis strains by 16S-23S spacer region based random amplified polymorphic DNA analysis. J Clin Microbiol 1995; 33: $1418-1420$

24. Haas WH, Butler WR, Woodley CL, Crawford JT. Mixed linker polymerase chain reaction: a new method for rapid fingerprinting of isolates of the Mycobacterium tuberculosis complex. J Clin Microbiol 1993; 31: 12931298.

25. Ross BC, Dwyer B. Rapid, simple method for typing isolates of Mycobacterium tuberculosis by using the polymerase chain reaction. J Clin Microbiol 1993; 31: 329-334.

26. Plikaytis BB, Crawford JT, Woodley CL, et al. Rapid amplification based fingerprinting of Mycobacterium tuberculosis. J Gen Microbiol 1993; 139: 1537-1542.

27. Goyal M, Young D, Zhang Y, Jenkins PA, Shaw RJ. PCR amplification of variable sequence upstream of kat $G$ gene to subdivide strains of Mycobacterium tuberculosis complex. J Clin Microbiol 1994; 32: 3070-3071.

28. Friedman CR, Stoeckle MY, Johnson WD Jr, Riley LW. Double-repetitive-element PCR method for subtyping Mycobacterium Tuberculosis clinical isolates. J Clin Microbiol 1995; 33: 1383-1384.

29. Patel S, Wall S, Saunders NA. Hemi-nested PCR for IS6110 fingerprinting of Mycobacterium tuberculosis strains. J Clin Microbiol 1996; 34: 1686-1690. 